\title{
La implementación de la herramienta Kahoot en el aula universitaria
}

\section{Rosa Pastor Villa.}

Escuela Técnica Superior de Arquitectura de Valencia, Universitat Politècnica de València, ropasvil@csa.upv.es

\begin{abstract}
The Bologna process has meant a methodological change in University Teaching, whose objective is to improve the teaching-learning process in a context enriched by the use of technologies, responding to students' requirements.

The objective of this Work is to present a learning experience based on the game implemented in the area of Architectural Constructions, in the first year of University teaching, to inform about the process, result and the opinions obtained from the students.
\end{abstract}

Key words: Innovation, Experience of learning, Active methodology, Role playing

\footnotetext{
Resumen

El Plan Bolonia ha supuesto un cambio metodológico en la Enseñanza Universitaria, cuyo objetivo es mejorar el proceso de enseñanza-aprendizaje en un contexto enriquecido por el uso de las tecnologías, respondiendo a los requerimientos de los estudiantes.

El objetivo de este Trabajo es presentar una experiencia de aprendizaje basada en el juego, implementada en el área de Construcciones Arquitectónicas, en el primer curso de enseñanza Universitaria, para informar sobre el proceso, resultado y las opiniones obtenidas del alumnado.
}

Palabras clave: Innovación, Experiencia de aprendizaje, Metodología activa, Role playing 


\section{Introducción}

La adaptación de los programas a las nuevas titulaciones de grado basadas en una docencia por competencias, implica un cambio sustancial en el enfoque de la enseñanza-aprendizaje y del rol profesor-alumno. En este marco de adaptación a las nuevas metodologías docentes, exponemos una práctica de aprendizaje basada en el uso de las Tic, mediante la implementación del juego en el aula, para facilitar y potenciar otros procesos de adquisición y puesta en práctica de conocimientos, que impliquen una participación activa del estudiante. Existen evidencias de su eficacia a través del empleo de herramientas de respuesta de audiencia (HRA) al introducir procesos de participación activa en el aula de manera sencilla y con un coste de mínimo de implementación, dado que muchos alumnos acuden a las clases con algún tipo de dispositivo móvil (teléfonos inteligentes, tabletas, portátiles, etc.) (Fuertes, Ariadna et al., 2016].

El Mobile learning es una nueva forma de educación que conjuga el e-learning y la utilización de los dispositivos móviles inteligentes. Es un valor añadido al proceso enseñanzaaprendizaje que permite la interconectividad y elimina cualquier dependencia de lugar o espacio. Dentro de este nuevo paradigma educativo destacamos la herramienta Kahoot (https://kahoot.it/), aplicación informática que permite la creación de cuestionarios en distintos formatos: quiz (preguntas), jumble (puzzle), discusión (debate) y survey (encuesta). Esta herramienta de gamificación del aula favorece el aprendizaje del alumnado por medio del juego, al tratarse de una experiencia motivadora.

Para crear un kahoot los pasos son sencillos, es necesario estar registrado en la web (https://create.kahoot.it/register) y entrar con uno de los perfiles que ofrece (a teacher, a student, socially, at work). La página permite, bien utilizar un repositorio de distintas materias publicados por otros usuarios, bien crear cuestionarios propios, adaptados a las necesidades específicas de la clase. El contenido de los cuestionarios puede incluir vídeos e imágenes, haciendo mucho más atractivo el juego.

Kahoot proporciona dos plataformas, una para el alumnado y otra para el profesorado; al inicio de cada sesión el sistema da una clave para que los concursantes se conecten a la actividad por medio de sus teléfonos móviles, tablets u ordenadores. Para empezar a jugar se proyectan las preguntas y los alumnos y alumnas por medio de sus dispositivos móviles contesta la opción que estimen correcta, teniendo en cuenta que a cada pregunta se le asigna un tiempo máximo de respuesta. Al finalizar cada alumno puede conocer su puntuación y se establece un ranking con las mismas. Después de cada cuestión se muestra la puntuación de los 3 mejores estudiantes, basándose tanto en el acierto de la pregunta como en la rapidez de respuesta.

La aplicación permite exportar los resultados a Excel o incluirlos en Google Drive, material que puede ser útil para el proceso de evaluación.

Al finalizar cada cuestionario se puede activar una breve encuesta acerca de la actividad, donde los estudiantes indican, con una valoración en una escala de 1 a 5, si han aprendido, si lo recomendarían y cómo se han sentido haciendo la actividad.

Implica por parte del profesorado:

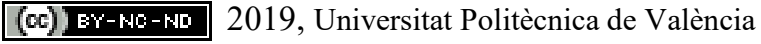


1. Seleccionar los contenidos.

2. Elaborar los cuestionarios que recojan dichos contenidos.

3. Tras obtener los resultados inmediatos, determinar qué contenidos no han quedado claros y deben ser reforzados

4. Ofrece retroalimentación a los alumnos en tiempo real, reflexiona con ellos sobre los resultados.

La experiencia educativa se ha realizado durante el curso 2018-19 en la asignatura Introducción a la Construcción que se imparte en el primer curso de Grado en Fundamentos de la Arquitectura, en la Escuela Técnica Superior de Arquitectura de Valencia (UPV). La asignatura nace del nuevo plan de estudios puesto en marcha en el curso 2002-2003. Los alumnos cuando cursan la asignatura se enfrentan por primera vez a contenidos relacionados con la construcción arquitectónica, el diseño de detalles constructivos y un amplio vocabulario específico que la mayoría desconocen.

\section{Objetivos}

El objetivo del presente trabajo es presentar una experiencia de gamificación en el aula universitaria utilizando el recurso informático Kahoot (Tortosa Ybáñez, M.T. y otros, 2016), realizada en el primer curso de Fundamentos de Arquitectura en la Universitat Politècnica de València. Posteriormente analizaremos la funcionalidad de la propuesta mediante el análisis de los resultados y la implementación de posibles mejoras para el curso 2019-20.

\section{Desarrollo de la innovación}

\subsection{Contexto}

El contexto en el que se desarrolla la innovación es el siguiente:

\begin{tabular}{|l|l|}
\hline \multicolumn{2}{|l|}{ EXPERIENCIA: GAMIFICACIÓN DEL AULA MEDIANTE KAHOOT } \\
\hline GRADO & FUNDAMENTOS DE ARQUITECTURA \\
\hline DEPARTAMENTO & CONSTRUCCIONES ARQUITECTÓNICAS \\
\hline ASIGNATURA & INTRODUCCIÓN A LA CONSTRUCCIÓN \\
\hline CARACTER & TRONCAL \\
\hline CRÉDITOS & 4,5 \\
\hline RATIO ALUMNOS/AULA & 50 \\
\hline
\end{tabular}

La materia impartida en la asignatura es la base para poder acometer la de los cursos superiores en los que se van ampliando los contenidos sobre la práctica constructiva en arquitectura, y es de especial relevancia para la formación del futuro arquitecto en el desarrollo de su profesión. 


\subsection{Metodología}

Para que las clases sean más dinámicas y motivadoras, al inicio de cada clase se realiza un cuestionario individual mediante la aplicación Kahoot, con los contenidos de la asignatura vistos en la sesión anterior.

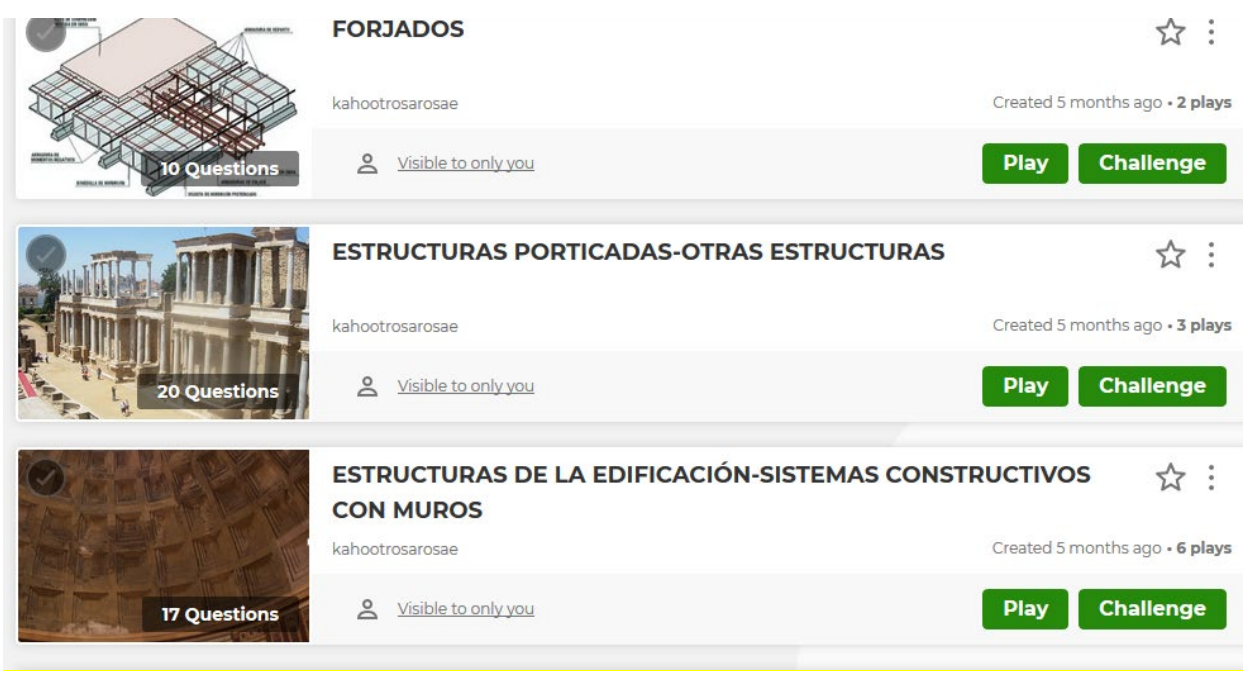

Fig. 1 Ejemplo de cuestionarios por temas de la asignatura

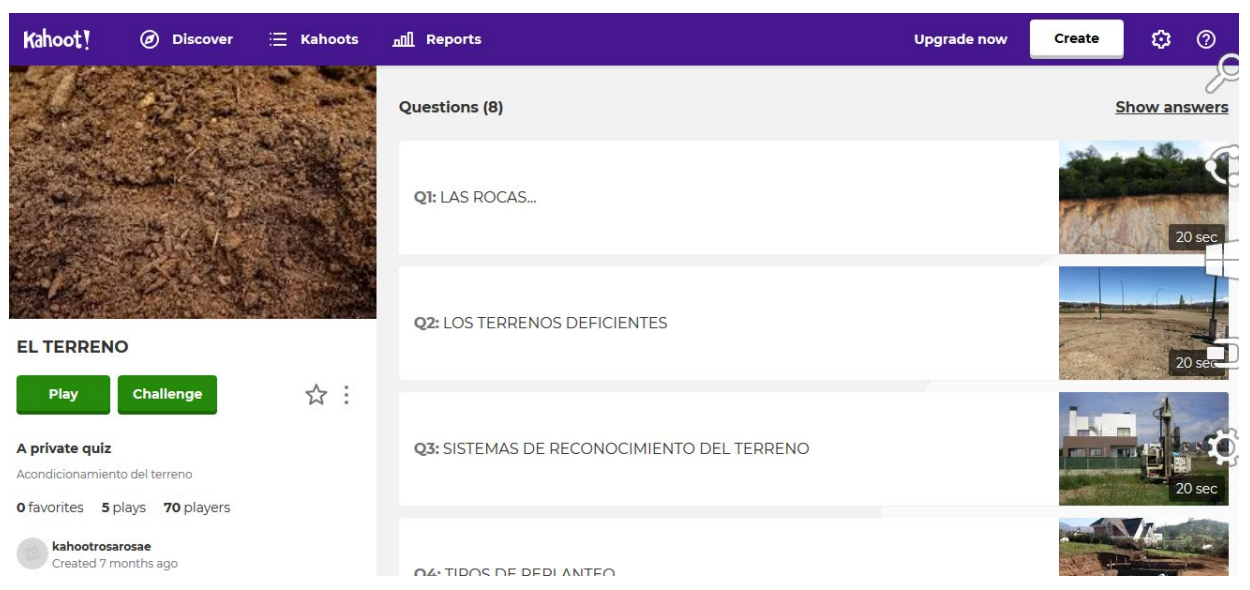

Fig. 2 Ejemplo de preguntas de uno de los temas de la asignatura

La experiencia consiste en :

- Breve explicación de la herramienta informática a los alumnos (primera sesión) 
- Se indica el número de preguntas de que consta el cuestionario y el tiempo asignado a cada pregunta

- Proyección en pantalla de la clave de conexión a la plataforma

- Comienza el cuestionario

- Las preguntas que no han quedado claras se refuerzan

- Análisis de los resultados

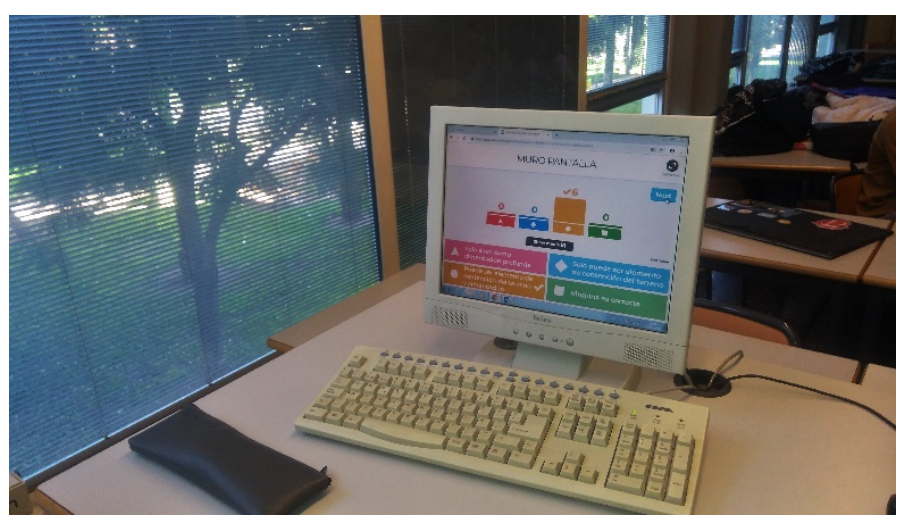

Fig. 3 Resultados de una pregunta de cuestinario mediante Kahoot

\section{Resultados}

La gamificación del aula mediante la implementación de la herramienta informática Kahoot ha sido muy satisfactoria para los alumnos y alumnas que han asistido regularmente a clase. Se observa respecto de años anteriores una mayor motivación reduciendo la tasa de abandono que suele ocurrir a final de curso.

Al final de curso se realizó un cuestionario entre el alumnado con dos preguntas abiertas sobre el empleo del Kahoot en la asignatura, para ver la calidad de la propuesta (Zabalza 2003-2004, 118) y su aceptación:

\section{Consideras que el Kahoot favorece el aprendizaje de la asignatura? Por qué?}

El $95 \%$ de las contestaciones fueron en sentido positivo y entre las justificaciones se extraen las más relevantes:

- Es una forma divertida de aprender, jugando se aprende sin darte cuenta

- Es una manera divertida de aprender y se te queda

- Es una manera diferente y divertida de aprender

- Favorece el aprendizaje y dinamiza las clases 
- Obliga a prestar atención a las preguntas, es interactivo diferente a escuchar y estar sentado, incita a participar

- Obliga a estudiar a diario, supone una rutina de estudio

- Te obliga a ejercitar lo aprendido en la clase anterior

- Es divertido competir para ser de los mejores

- Es una manera más dinámica de aprender, compitiendo se aprende más rápido

- Es una forma de aprender y competir

- Ayuda a pensar en las clases teóricas

- Anima a estudiar cada día un poco más

- Me motiva para aprender

- Repasamos de una manera divertida

- El método es entretenido y eficaz

- Aprendemos de los errores

El 5\% de las opiniones en sentido negativo de los alumnos se destaca:

- Importa mucho la rapidez de contestación y a veces estudias mucho y no obtienes los resultados deseados

- Es una pérdida de tiempo y no valora realmente el esfuerzo de estudiar

- Se puede intuir el mecanismo de las preguntas (todas son correctas)

- Cuenta mucho la suerte y la intuición

- Es un método injusto porque se copian. No sirve

\section{2. ¿Qué mejoras plantearías?}

- Aumentar el número de preguntas engañosas, para pensar

- Incorporar más preguntas

- Incrementar el tiempo de respuesta

- Eliminar la opción todas son correctas

- Implementarlo en mas asignaturas

- Realizarlo en grupo

Del análisis de las encuestas se deduce que el método de aprendizaje ha sido valorado de manera muy positiva por la mayor parte del alumnado

\section{Conclusiones}

Para que la implementación de la innovación ofrezca los resultados esperados es necesario que los dos agentes del proceso de enseñanza-aprendizaje, profesorado y alumnado, se impliquen. La experiencia planteada ha motivado tanto al docente de la asignatura como a los alumnos y alumnas. 
Respecto del aprendizaje del alumnado se aprecian las siguientes ventajas del método:

- Llevan al día el temario de la asignatura de construcción con entusiasmo, y deben realizar un esfuerzo menor cara al examen, obteniendo mejores resultados.

- Aumenta su motivación respecto al contenido estudiado.

- Están más activos en su aprendizaje.

- Realizan un aprendizaje más significativo.

- Adquieren competencias transversales.

Respecto del trabajo del profesor, al haberse implementado la experiencia por primera vez en la asignatura ha supuesto un trabajo extra para su diseño y coordinación de las actividades dentro de clase, y selección para la elaboración del material docente (Marin-García J.A, 2007), pero este trabajo previo sirve de base para los siguientes cursos, en los que se plantean las mejoras expuestas en apartado anterior.

\section{Referencias}

MARIN-GARCIA, J. A. (2007). Conversión de una asignatura hacia metodologías activas. carga de trabajo de alumnos y profesores. Revista Alternativas - Serie: Espacio Pedagogico $\underline{12(46 / 47): 51-60 .}$

ZABALZA, M.A. (2003-2004). Innovación en la enseñanza universitaria, en Contextos educativos. 6-7, pp 113-136

Tortosa Ybáñez, María Teresa; Grau Company, Salvador; Álvarez Teruel, José Daniel (coords.). XIV Jornades de Xarxes d'Investigació en Docència Universitària. Investigació, innovació i ensenyament universitari: enfocaments pluridisciplinaris $=$ XIV Jornadas de Redes de Investigación en Docencia Universitaria. Investigación, innovación y enseñanza universitaria: enfoques pluridisciplinares. Alacant: Universitat d'Alacant, Institut de Ciències de l'Educació, 2016. ISBN 978-84-608-7976-3, pp. 1241-1254

Fuertes, Ariadna [et al.]. Uso de herramientas de respuesta de audiencia en la docencia presencial universitaria. Un primer contacto.. A: XXII Jornadas de Enseñanza Universitaria de la Informática. "Actas de las XXII JENUI". Universidad de Almería, 2016, p. 261268. http://hdl.handle.net/2117/90357 . Depósito legal 1007-2016 